Research Article

\title{
Determination of Genetic and Epigenetic Modifications-Related Prognostic Biomarkers of Breast Cancer: Genome High-Throughput Data Analysis
}

\author{
Chundi Gao $\mathbb{D}^{1},{ }^{1}$ Huayao Li $\mathbb{D}^{2},{ }^{2}$ Cun Liu ${ }^{D},{ }^{1}$ Jibiao Wu $\mathbb{D}^{2},{ }^{2}$ Chao Zhou $\mathbb{D}^{3},{ }^{3}$ Lijuan Liu $\mathbb{D}^{3},{ }^{3}$ \\ Jing Zhuang $\mathbb{D}^{3},{ }^{3}$ and Changgang Sun $\mathbb{D}^{3,4}$ \\ ${ }^{1}$ College of First Clinical Medicine, Shandong University of Traditional Chinese Medicine, Jinan, Shandong 250014, China \\ ${ }^{2}$ College of Basic Medical, Shandong University of Traditional Chinese Medicine, Jinan, Shandong 250014, China \\ ${ }^{3}$ Department of Oncology, Weifang Traditional Chinese Hospital, Weifang, Shandong 261041, China \\ ${ }^{4}$ Qingdao Academy of Chinese Medical Sciences, Shandong University of Traditional Chinese Medicine, Qingdao, China
}

Correspondence should be addressed to Jing Zhuang; 13963676719@163.com and Changgang Sun; scgdoctor@126.com

Received 30 April 2021; Revised 11 August 2021; Accepted 26 August 2021; Published 14 September 2021

Academic Editor: Yuan Seng Wu

Copyright (c) 2021 Chundi Gao et al. This is an open access article distributed under the Creative Commons Attribution License, which permits unrestricted use, distribution, and reproduction in any medium, provided the original work is properly cited.

The high heterogeneity of breast cancer (BRCA) makes it more challenging to interpret the genetic variation mechanisms involved in BRCA pathogenesis and prognosis. Areas with high DNA methylation (such as CpG islands) were accompanied by copy number variation $(\mathrm{CNV})$, and these genomic variations affected the level of DNA methylation. In this study, we characterized intertumor heterogeneity and analyzed the effects of CNV on DNA methylation and gene expression. In addition, we performed a Genetic Set Enrichment Analysis (GSEA) to identify key pathways for changes between patients with low and high expression of genes. Our analysis found two key genes, namely, HPDL and SOX17. The protein expressed by HPDL is 4-hydroxyphenylpyruvate dioxygenase-like protein, which has dioxygenase activity. SOX17 is a transcription factor that can inhibit Wnt signaling, promote the degradation of activated CTNNB1, and participate in cell proliferation. Our analysis found that the CNV of HPDL and SOX17 is not only related to the patient's prognosis, but also related to gene methylation and expression levels affecting the patient's survival time. Among them, the high-methylation, low-expression HPDL and SOX17 showed poor prognosis. And the addition of two copies of SOX17 is associated with a lower survival rate, while a decrease in the copy number of HPDL also suggests a poor prognosis. This study provided an effective bioinformatics basis for further exploration of molecular mechanisms related to BRCA and assessment of patient prognosis, but the development of biomarkers for diagnosis and treatment still requires further clinical data validation.

\section{Introduction}

In the postgenomic era, rapidly evolving high-throughput sequencing technologies have enabled the acquisition of vast amounts of multiomics data more efficiently [1]. The variation of expression of some genes causes the genetic regulation trajectory inside the cell to deviate, which alters the gene expression programming inside the cell. Therefore, most disease-causing genomic variants are likely to play a role by altering gene regulation, such as transcription factor binding and DNA methylation, rather than directly affecting protein function $[2,3]$. The high heterogeneity of breast cancer (BRCA) makes it more challenging to interpret the genetic variation mechanisms involved in BRCA pathogenesis and prognosis [4].

In human cancer, genomic instability leads to extensive cell copy number variation (CNV) [5]. Genome-wide association studies (GWASs) have been conducted for common malignancies and have identified more than 450 genetic variants associated with increased disease risk [6]. In BRCA, $\mathrm{CNV}$ is associated with about $40 \%$ of gene expression, which can participate in the occurrence and development of BRCA and affect the prognosis of patients [7]. It has been found that changes in CNV such as BRCA1, BLM, and OR4C11 
will increase the incidence of BRCA. BRCA1, BLM, and OR4C11 are all related to cell proliferation. BRCA1 is a transcriptional activator that can regulate the cell cycle; BLM is involved in DNA replication and repair, and OR4C11 can regulate cell signal transduction [8], while changes in $\mathrm{CNV}$ such as MYC and JAK2 play a role in acquired chemotherapy resistance to triple-negative BRCA [9]. In addition, the higher intratumoral heterogeneity of EGFR/CEP7 and CCND1/CEP11 CNV could predict metastasis and was significantly correlated with metastasis-free survival in triple negative BRCA patients [10].

Disorders in the epigenetic state are closely related to human diseases, particularly cancer. DNA methylation is a well-characterized epigenetic modification that is closely related to many cellular processes. In the current study, DNA methylation and its sites associated with tumor recurrence and overall survival (OS) of BRCA and its subtypes have been identified based on methods employed for genome-wide DNA methylation analysis [11-13]. The methylation of oncogenes, ESR1 and ERBB2, and tumor suppressor genes, FBLN2, CEBPA, and FAT4, contribute to the early diagnosis of BRCA [14]. And the methylation of HER2, Ki67, and GSTP1 are associated with BRCA TNM staging and tumor size and can be combined for early diagnosis and prognosis [15].

$\mathrm{CNV}$ represents a major source of genomic variation and is an important genetic factor leading to various cancers. DNA methylation, a major means of epigenetic modification, is considered an inhibitory epigenetic marker. Several studies have found that areas with high DNA methylation (such as CpG islands) are accompanied by copy number variation, and these genomic variations affect the level of DNA methylation [16]. For example, in lung adenocarcinoma, DNA methylation heterogeneity demonstrates branch clonal evolution of lung adenocarcinoma regions driven by genomic instability and subclone copy number variation [17]. Here, we investigated the association between genomic variation (such as $\mathrm{CNV}$ ) in regulatory regions of BRCA and corresponding changes in DNA methylation. In addition, we performed a Genetic Set Enrichment Analysis (GSEA) to identify key pathways for changes between patients with low and high expression of genes. Thus, an indepth study of the genome pathogenesis of BRCA was conducted to identify prognostic biomarkers and their clinical efficiency.

\section{Materials and Methods}

2.1. Data Processing and Analysis. The BRCA-related methylation, $\mathrm{CNV}$, gene expression, and clinical data were downloaded from The Cancer Genome Atlas (TCGA) GDC (https://gdc.cancer.gov/). The chi-square test and Limma and edgeR software packages were used to collate and analyze the downloaded data and screened according to $P$ and $\operatorname{logFC}$ values. To obtain differences in CNV, abnormally methylated and dysregulated genes between BRCA tissue samples and normal tissue samples were analyzed. The data from the TCGA database is public. Therefore, no approval from the local ethics committee was required.
2.2. Multilayer Correlation Analysis Predicts the Pattern of Gene CNV in BRCA. DNA methylation has been shown to regulate gene expression in a variety of ways, such as changing chromosome structure, DNA stability, etc. In addition, CNV is widely distributed in the human genome and has important biological implications. To further explore the link between CNV and methylation on gene expression, the possible patterns of CNV in BRCA need to be elucidated. This study focuses on the analysis of correlation between abnormal methylation and gene expression, $\mathrm{CNV}$ and aberrant methylation, and $\mathrm{CNV}$ and gene expression. Screening was done by the Pearson correlation coefficient and $P$ value. Key genes with simultaneous methylation abnormalities, CNV, and abnormal expression were obtained, and further prognostic analysis was performed on these genes.

2.3. Mapping of Kaplan-Meier Survival Curve of Genes and Screening of Prognostic Key Genes. In order to further identify key genes related to the prognosis of BRCA patients from the genes obtained above, survival analysis was performed on the relevant data based on the survival software package, and survival curves were plotted to show the effect of abnormal methylation and methylation combined with abnormal gene expression on patient survival. In addition, in order to further explore the methylation sites of prognostic aberrant methylation genes, the factors affecting the prognosis of patients and gene expression are mapped to specific methylation sites.

\subsection{The Impact of CNV of Key Genes on Patient Prognosis.} Through data analysis, it was found that the abnormal methylation of key genes is closely related to the prognosis of BRCA patients, while the key genes harbored methylation abnormalities, $\mathrm{CNV}$, and abnormal expression, and there was a significant correlation between them. The effect of mutations on the prognosis of patients can be seen by studying CNV and survival time of BRCA patients, further indicating the biological significance of gene $\mathrm{CNV}$ in the progression of BRCA. In addition, we performed GSEA analysis between high-expression and low-expression groups of key genes to determine key pathways that are altered in patients with abnormal gene expression [18].

\section{Results}

3.1. Data Processing and Analysis. In this study, BRCArelated methylation data downloaded from the TCGA database included 883 samples, comprising 96 normal tissue samples and 787 BRCA tissue samples. The difference analysis results obtained a total of 122 proteincoding genes with $P<0.05$ and $|\log \mathrm{FC}|>1$ as the cutoff condition (Figure 1(a)). The CNV data included 2201 samples, 1103 normal tissue samples, and 1098 BRCA tissue samples. A total of 19178 genes with $\mathrm{CNV}$ were found based on the chi-square test results $(P<0.05)$ 


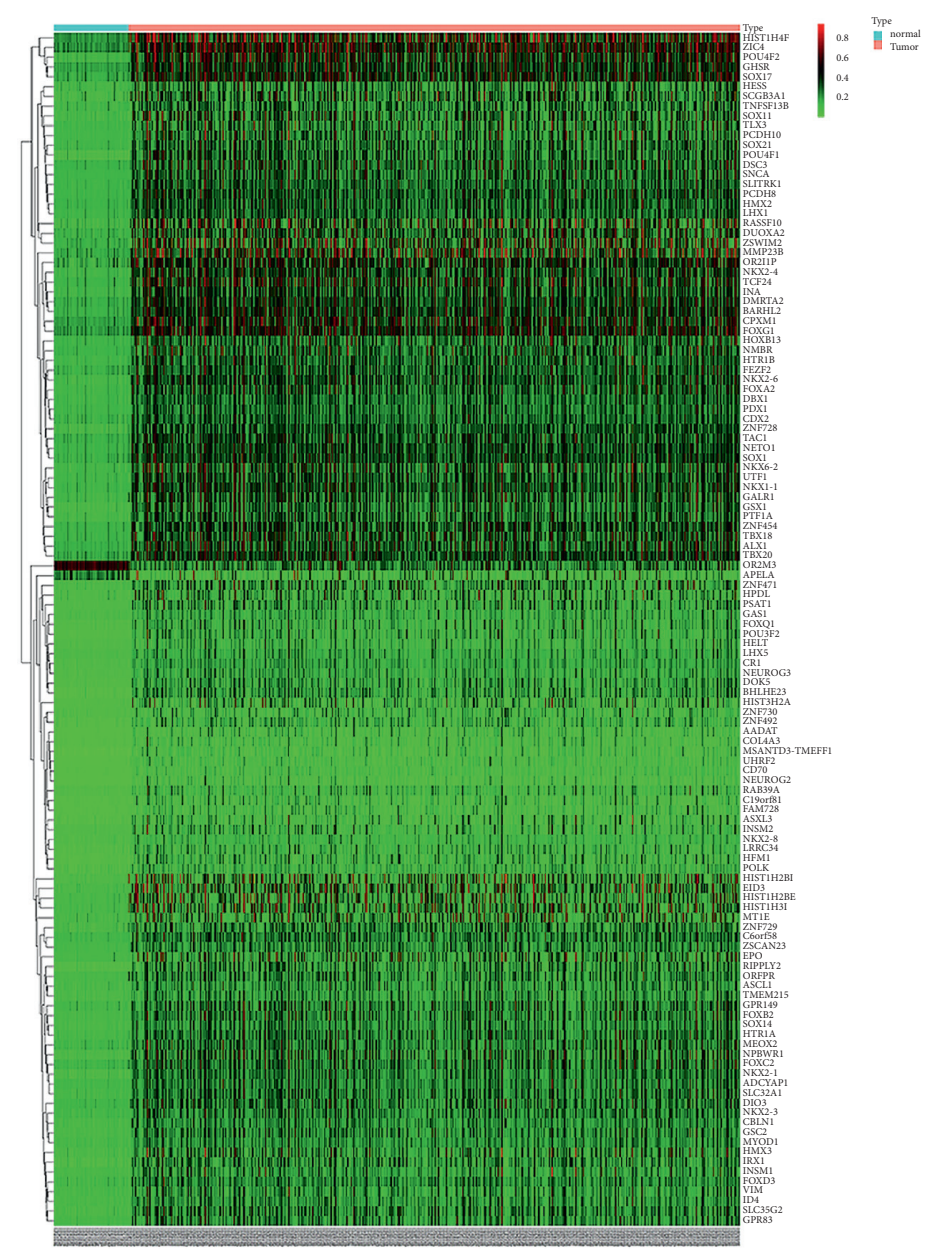

(a)

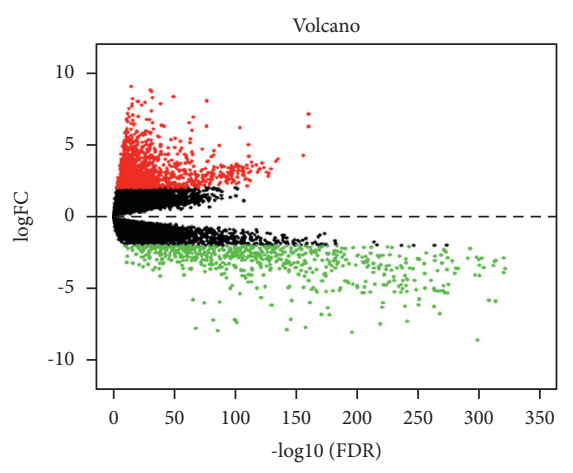

(b)

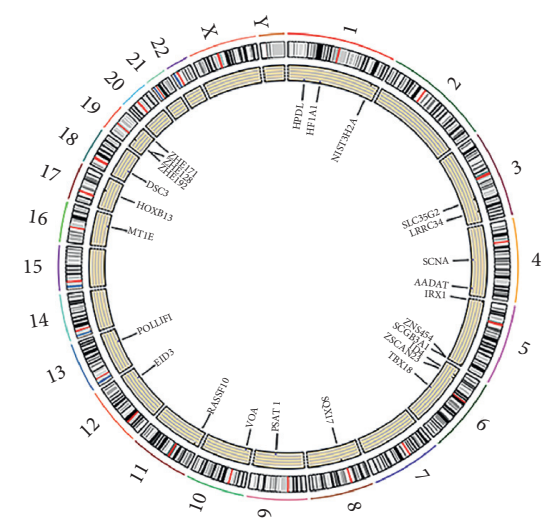

(c)

Figure 1: (a) The heat map of BRCA-related aberrant methylated genes. The color from green to red shows a trend from low expression to high expression. (b) The volcano diagram of BRCA-related differentially expressed genes. The red dot represents upregulated genes, and green dot represents downregulated genes. (c) The CNV circle map of BRCA-related genes on chromosome. The points at the periphery indicate copy number amplification, and the points at the inner circumference indicate a decrease in copy number.

(Supplementary Material 1). The difference analysis of gene expression data between 112 normal tissue samples and 1096 cancer tissue samples showed that 2138 dysregulated genes, including 1375 upregulated genes and 763 down regulated genes (Figure $1(\mathrm{~b})$ ), were obtained with $P<0.01$ and $|\log \mathrm{FC}|>2$ as the cutoff condition.
3.2. Multilayer Correlation Analysis to Screen Key Genes. In order to reduce the number of calculations of correlation analysis between the two, we performed correlation analysis on the condition of genes with abnormal methylation. First, we found that 105 of the 122 genes with aberrant methylation exhibited simultaneous expression 
disorders. Combining methylation and expression-related samples (857 in total) for correlation analysis showed that the aberrant methylation of 25 genes was closely related to the expression with the Pearson correlation coefficient Cor $>0.4$ as the screening criterion (Table 1). Interestingly, these 25 genes harbored CNV simultaneously (Figure 1(c)). To explore the pattern of effects of CNV in disease progression, we performed a correlation analysis of CNV with methylation and abnormal gene expression for 25 genes. Among them, CNV and methylation-related samples were combined with a total of 855 , and CNV and expressionrelated samples were combined with a total of 1172 . Screening with $P<0.01$ as the cut-off criterion, the CNV of 12 genes was associated with the level of methylation, and the $\mathrm{CNV}$ of 16 genes was related to the abnormal expression level. Among them, there are 6 common genes (Figure 2). We used these six genes as key genes for prognostic survival analysis.

3.3. Joint Survival Analysis and Site-Related Prognostic Assessment to Identify Biomarkers. Through joint survival analysis, it was found that the combination of methylation and abnormal expression of HPDL and SOX17 was significantly associated with the prognosis of BRCA patients. Furthermore, the results showed that high-methylation lowexpression of HPDL and SOX17 showed poor prognosis (Figure 3(a)). In addition, based on the survival of the $\mathrm{R}$ package, we analyzed the effects of the relevant methylation sites of these two genes on patient survival. $P<0.05$ was used as a screening criterion for predicting prognosis, and specific methylation sites associated with the prognosis of these genes were found. Among them, the two methylation sites of HPDL and the eight methylation sites of SOX17 can affect the survival time of patients (Figure 3(b)).

3.4. Kaplan-Meier Survival Curve Analysis of the Effect of Gene CNV on Patient Prognosis. The genes HPDL and SOX17 showed not only methylation abnormalities and abnormal expression, but also CNV. Further analysis showed that CNV in HPDL and SOX17 were associated with overall patient survival, in which the addition of two copies of SOX17 is associated with a lower survival rate, while a decrease in the copy number of HPDL also suggests a poor prognosis (Figure $3(\mathrm{c})$ ). In addition, as the CNV of HPDL and SOX17 are related to methylation and abnormal expression levels, our research indicated that the CNV of HPDL and SOX17 can directly affect the prognosis of patients and can also indirectly affect the survival time of patients by affecting the methylation and expression levels of the corresponding genes.

3.5. GSEA Analysis of Patients with Low and High Expression of HPDL and SOX17. To identify the molecular pathways of the biological functions and effects of HPDL and SOX17 in BRCA progression, we used GSEA to identify key pathways involved in the changes between patients with low and high expression of genes. With $P$ value $<0.05$ as the screening standard, the results indicated that the pathways that HPDL can affect mainly, including MAPK signaling pathway and p53 signaling pathway. In addition, SOX17 mainly affects JAK-STAT signaling pathway, WNT signaling pathway, and so on (Table 2, Figure 4).

\section{Discussion}

Heterogeneity is an important predictor of tumor treatment failure and drug resistance, and genomic mutations (such as copy number variation) are important causal factors of heterogeneity among tumors. Previous studies have shown that CNV can affect the expression level of proteins through epigenetic regulation, and the key mechanism is to affect epigenetic modifications (such as DNA methylation). The overall hypomethylation of oncogenes and hypermethylation of tumor suppressor genes are characteristic of most cancer types. Molecular understanding of BRCA heterogeneity is the key to effective treatment and personalized medicine. In this study, we used TCGA highthroughput molecular profiling data to characterize intertumor heterogeneity and analyzed the effects of $\mathrm{CNV}$ on DNA methylation and gene expression.

In our analysis, CNV of HPDL and SOX17 affected methylation and gene expression levels in BRCA, and CNV and methylation of HPDL and SOX17 can lead to poor prognosis in patients with BRCA. In this study, it was found that the CNV of SOX17 showed copy number amplification on chromosome 8, while the CNV of HPDL showed a decrease in copy number on chromosome 1. Further analysis showed that when the copy number of SOX17 increased or the copy number of HPDL decreased, the prognosis of BRCA patients was poor. The CNV of SOX17 and HPDL can affect the expression of genes through epigenetic modification, and DNA methylation is an important pathway for epigenetic modification. The methylation sites of SOX17 that we characterized with BRCA OS included cg00123055, cg02222728, cg03329976, cg08044907, cg15377283, cg24150172, cg24891539, and cg24928317. The methylation sites of HPDL included cg12178578 and cg15071854. Survival analysis showed that the OS of BRCA patients hypermethylated in SOX17 and HPDL was poorer. Therefore, CNV and methylation of SOX17 and HPDL could predict recurrence, metastasis, and prognosis of BRCA patients.

SOX17, a transcriptional regulator, binds to target promoter DNA and inhibits Wnt signaling. SOX17 gene promoter methylation can be used as a tumor suppressor and dysregulated oncogene in many tumors [19-21]. In BRCA, Fu et al. used methylation-specific polymerase chain reaction to assess the relationship between the methylation of the SOX17 gene promoter and the onset and prognosis of BRCA. Abnormal SOX17 methylation in cancer tissues and plasma DNA was found to be significantly associated with tumor lymph node metastasis and lymph node metastasis, associated with poor disease-free survival $(P<0.005)$ and overall survival $(P<0.005)$. In addition, SOX17 methylation in plasma DNA is an independent prognostic factor for DFS in BRCA [22]. Chimonidou et al. found that the SOX17 
TABLE 1: 25 genes with significant correlation between methylation level and expression.

\begin{tabular}{|c|c|c|}
\hline Gene & Cor & $P$ value \\
\hline HOXB13 & 0.455 & $5.47 E-45$ \\
\hline SCGB3A1 & -0.404 & $5.75 E-35$ \\
\hline POU4F1 & -0.411 & $2.76 E-36$ \\
\hline SOX17 & -0.412 & $2.06 E-36$ \\
\hline SLC35G2 & -0.417 & $2.56 E-37$ \\
\hline RASSF10 & -0.421 & $4.45 E-38$ \\
\hline AADAT & -0.422 & $2.84 E-38$ \\
\hline HFM1 & -0.424 & $1.23 E-38$ \\
\hline HPDL & -0.426 & $3.82 E-39$ \\
\hline SNCA & -0.427 & $2.85 E-39$ \\
\hline TBX18 & -0.429 & $1.18 E-39$ \\
\hline VIM & -0.475 & $1.99 E-49$ \\
\hline DSC3 & -0.503 & $4.94 E-56$ \\
\hline LRRC34 & -0.505 & $1.23 E-56$ \\
\hline ZSCAN23 & -0.53 & $2.24 E-63$ \\
\hline ZNF454 & -0.538 & $2.08 E-65$ \\
\hline ZNF728 & -0.545 & $1.39 E-67$ \\
\hline MT1E & -0.566 & $9.90 E-74$ \\
\hline IRX1 & -0.567 & $4.62 E-74$ \\
\hline ZNF492 & -0.584 & $1.55 E-79$ \\
\hline PSAT1 & -0.587 & $1.64 E-80$ \\
\hline EID3 & -0.599 & $1.23 E-84$ \\
\hline ID4 & -0.605 & $1.26 E-86$ \\
\hline HIST3H $2 \mathrm{~A}$ & -0.658 & $2.66 E-107$ \\
\hline ZNF471 & -0.662 & $3.79 E-109$ \\
\hline
\end{tabular}

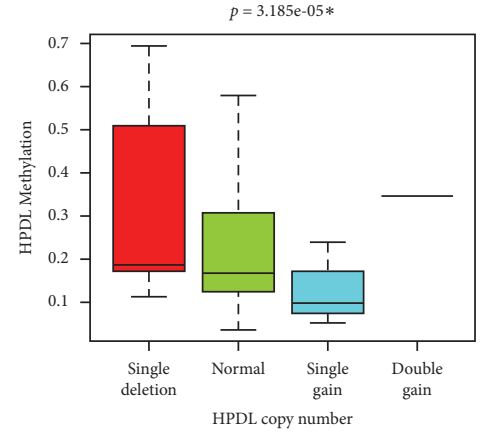

(a)

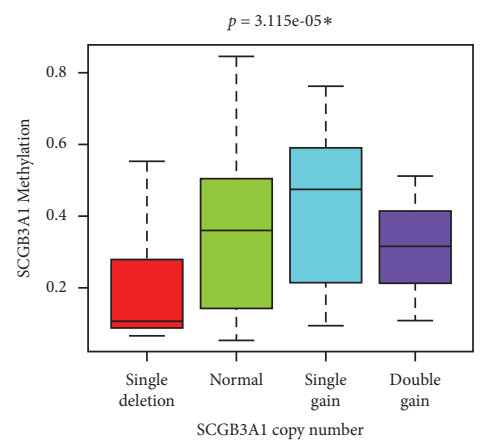

(d)

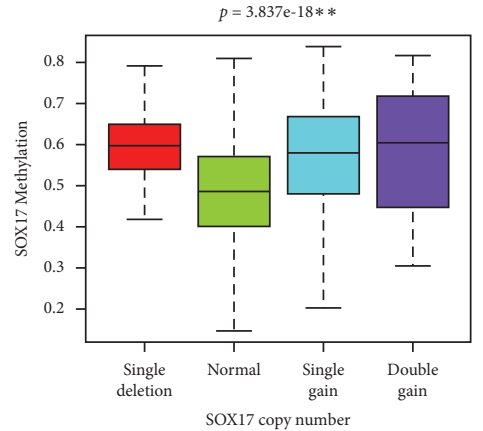

(b)

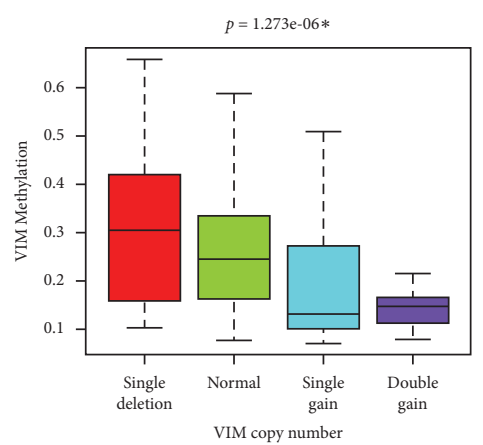

(e)

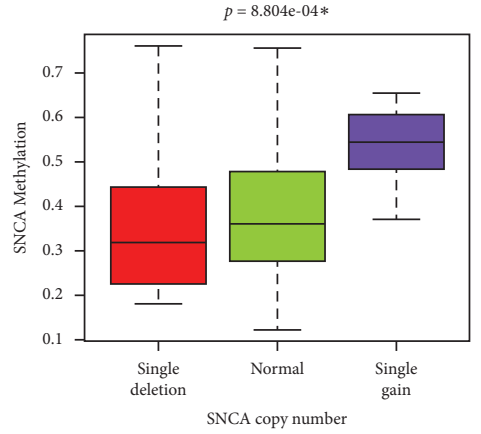

(c)

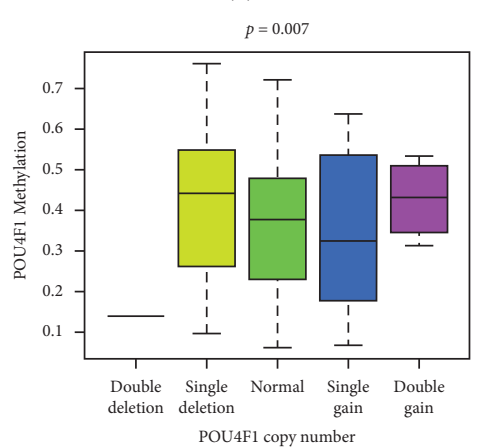

(f)

Figure 2: Continued. 


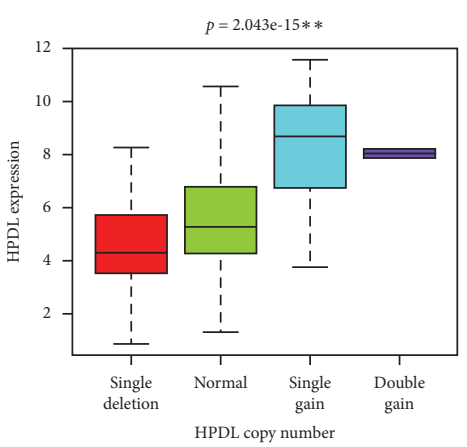

(g)

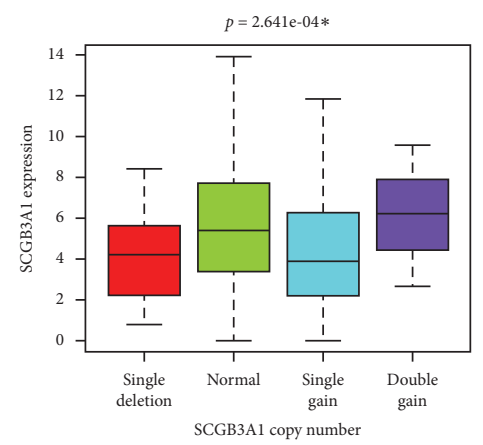

(j)

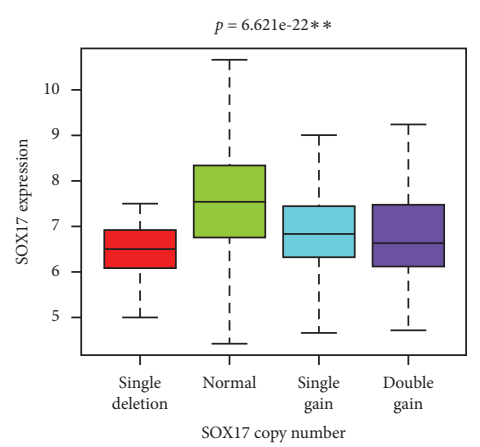

(h)

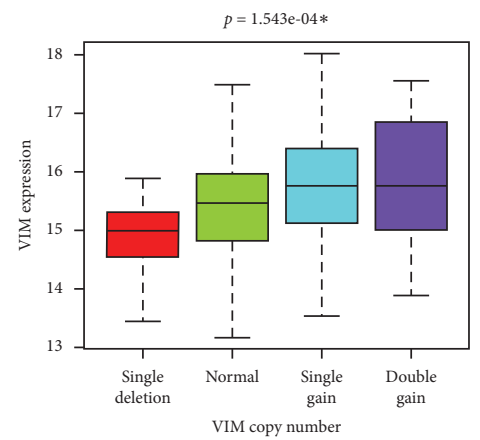

(k)

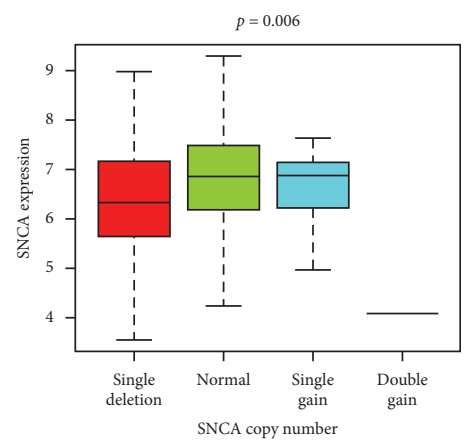

(i)

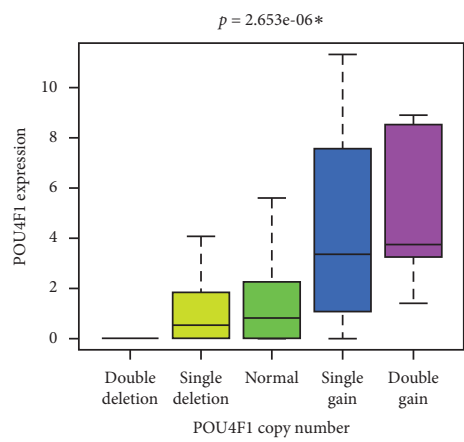

(l)

FIGURE 2: Six genes with CNV mutations that are related to both methylation and gene expression levels. (a-f) CNV and methylation. (g-l) $\mathrm{CNV}$ and expression.

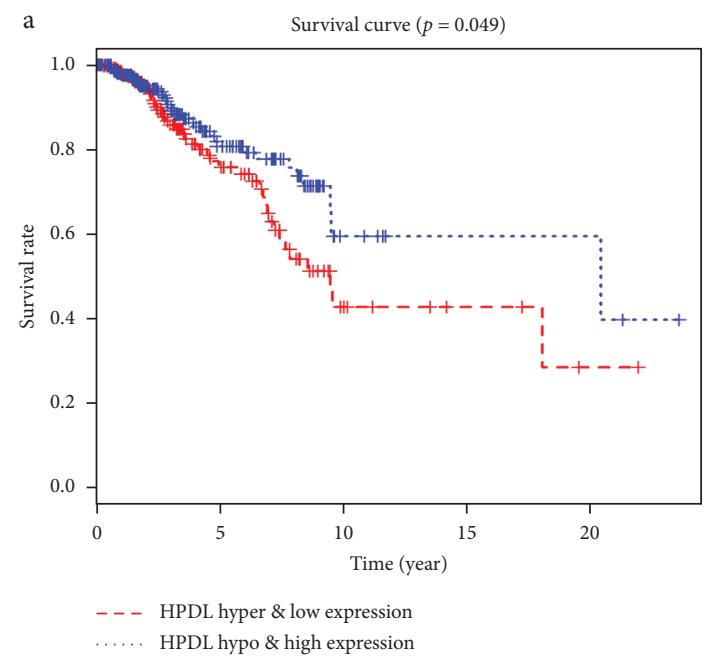

b

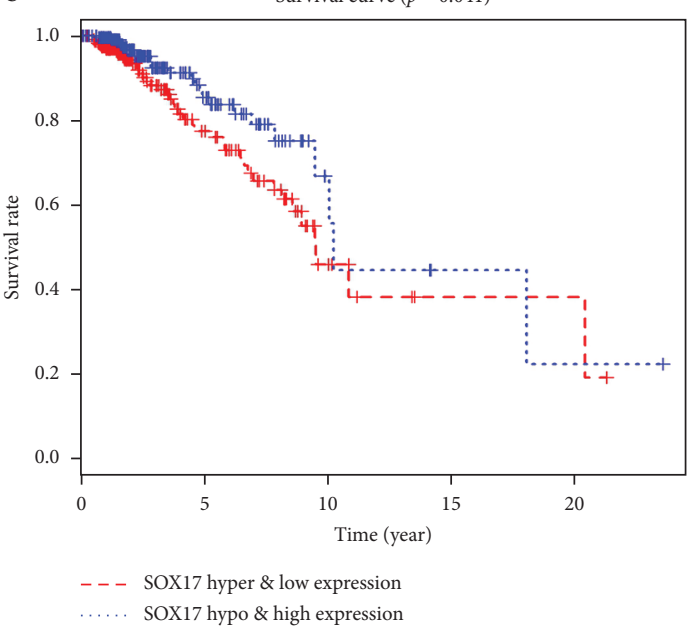

(a)

Figure 3: Continued. 

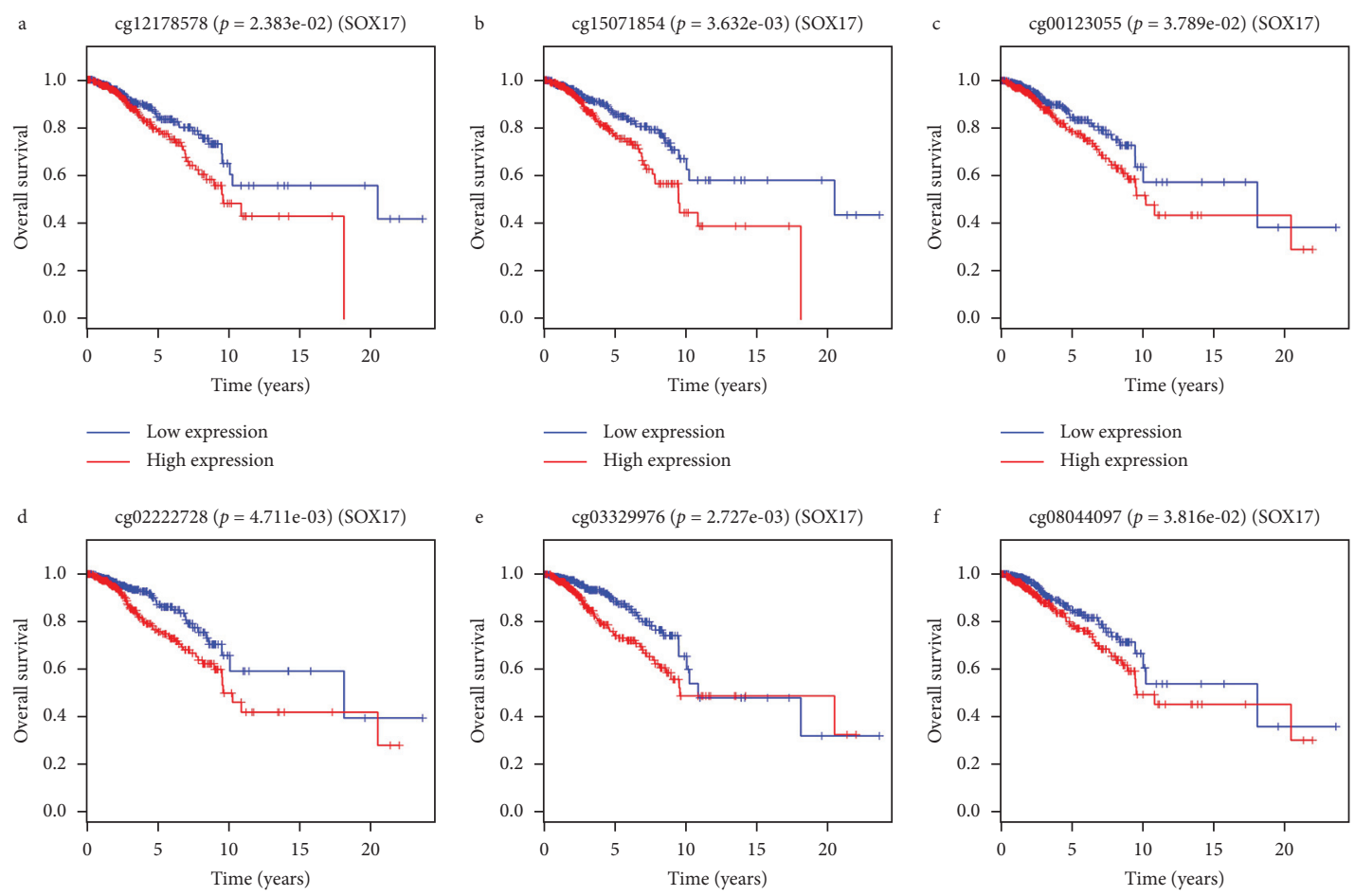

$$
\text { L_ Low expression }
$$

—_ Low expression

_ High expression

__ High expression
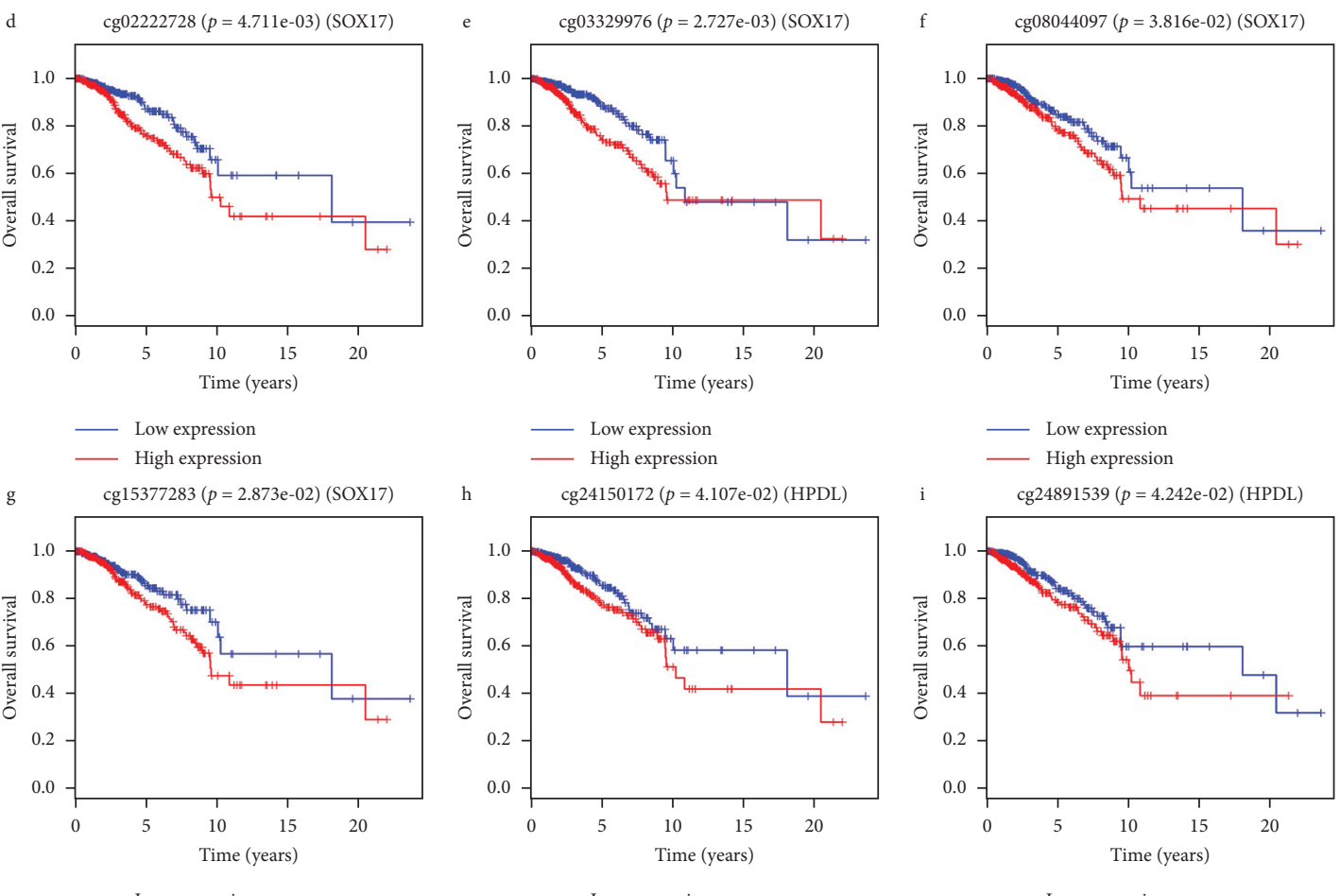

_ Low expression

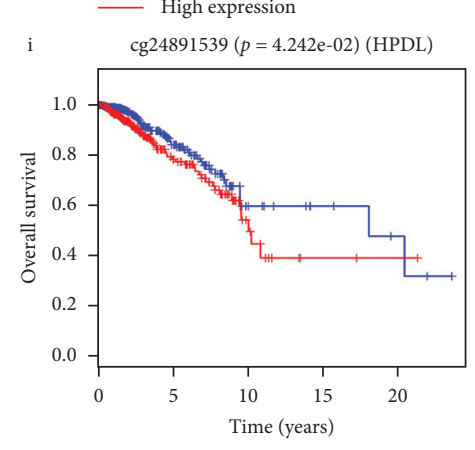

ow expression

L_ Low expression

_ Low expression

High expression

$$
\text { j }
$$

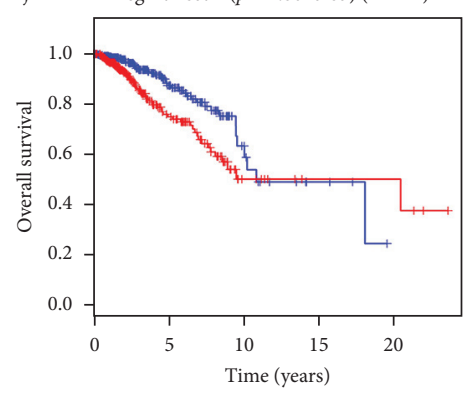

Low expression

- High expression

(b)

Figure 3: Continued. 

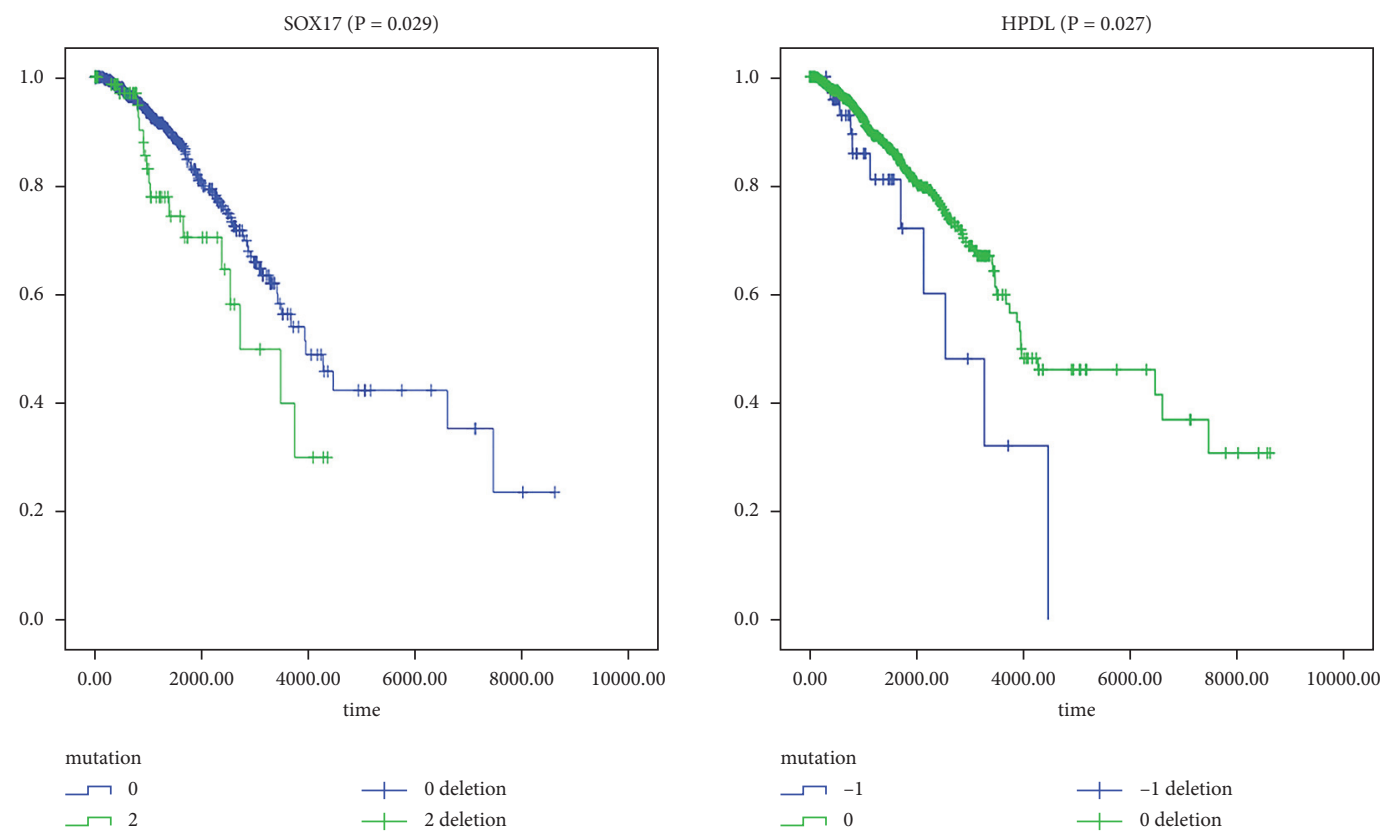

(c)

FIgURE 3: (a) Kaplan-Meier survival curves for the joint survival analysis. (A) The combination of gene HPDL methylation and expression; (B) the combination of gene SOX17 methylation and expression. (b) Kaplan-Meier survival curves of the related methylated sites. (A, B) Methylated sites of the gene HPDL; (C-J) methylated sites of the gene SOX17. (c) Kaplan-Meier survival curves for the copy number variation.

TABle 2: The key pathways for the differential between low and high expression of patients based on GSEA analysis.

\begin{tabular}{|c|c|c|c|}
\hline Gene & Name & NES & $P$ value \\
\hline \multirow{14}{*}{ HPDL } & KEGG_FOCAL_ADHESION & -1.91198 & $P<0.001$ \\
\hline & KEGG_DILATED_CARDIOMYOPATHY & -1.61559 & 0.002 \\
\hline & KEGG_HYPERTROPHIC_CARDIOMYOPATHY_HCM & -1.64029 & 0.002075 \\
\hline & KEGG_ADHERENS_JUNCTION & -1.74735 & 0.002283 \\
\hline & KEGG_ALDOSTERONE_REGULATED_SODIUM_REABSORPTION & -1.70915 & 0.002387 \\
\hline & KEGG_ARRHYTHMOGENIC_RIGHT_VENTRICULAR_CARDIOMYOPATHY_ARVC & -1.75006 & 0.006993 \\
\hline & KEGG_ABC_TRANSPORTERS & -1.5166 & 0.014141 \\
\hline & KEGG_MAPK_SIGNALING_PATHWAY & -1.66251 & 0.016713 \\
\hline & KEGG_TGF_BETA_SIGNALING_PATHWAY & -1.52333 & 0.04008 \\
\hline & KEGG_OOCYTE_MEIOSIS & 1.754743 & $P<0.001$ \\
\hline & KEGG_PROGESTERONE_MEDIATED_OOCYTE_MATURATION & 1.685132 & $P<0.001$ \\
\hline & KEGG_CELL_CYCLE & 1.639552 & $P<0.001$ \\
\hline & KEGG_P53_SIGNALING_PATHWAY & 1.51856 & 0.035503 \\
\hline & KEGG_OLFACTORY_TRANSDUCTION & 1.609742 & 0.046472 \\
\hline \multirow{13}{*}{ SOX17 } & KEGG_OOCYTE_MEIOSIS & -1.58789 & 0.04814 \\
\hline & KEGG_JAK_STAT_SIGNALING_PATHWAY & 1.797007 & $P<0.001$ \\
\hline & KEGG_NEUROACTIVE_LIGAND_RECEPTOR_INTERACTION & 1.865758 & 0.001481 \\
\hline & KEGG_ABC_TRANANSPORTERS & 1.455277 & 0.014463 \\
\hline & KEGG_RETINOL_METABOLISM & 1.741166 & 0.017483 \\
\hline & KEGG_HEMATOPOIETIC_CELL_LINEAGE & 1.564893 & 0.021195 \\
\hline & KEGG_VASCULAR_SMOOTH_MUSCLE_CONTRACTION & 1.562236 & 0.023636 \\
\hline & KEGG_ADIPOCYTOKINE_SIGNALING_PATHWAY & 1.588887 & 0.029297 \\
\hline & KEGG_INSULIN_SIGNALING_PATHWAY & 1.672106 & 0.038113 \\
\hline & KEGG_METABOLISM_OF_XENOBIOTICS_BY_CYTOCHROME_P450 & 1.663495 & 0.039587 \\
\hline & KEGG_WNT_SIGNALING_PATHWAY & 1.528532 & 0.04 \\
\hline & KEGG_DRUG_METABOLISM_CYTOCHROME_P450 & 1.675274 & 0.043328 \\
\hline & KEGG_AXON_GUIDANCE & 1.497392 & 0.047445 \\
\hline
\end{tabular}




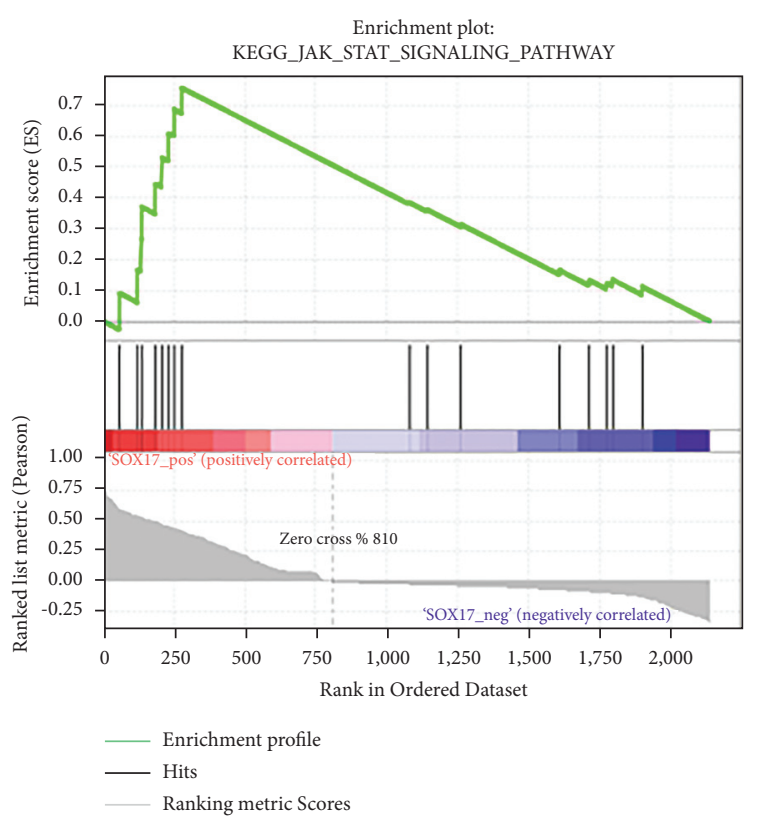

(a)

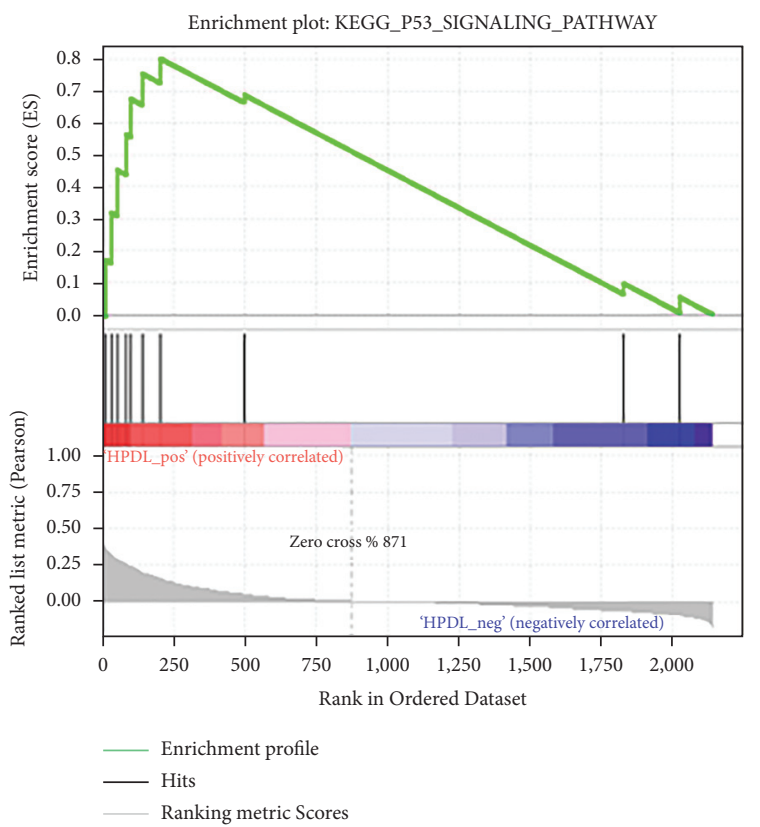

(c)

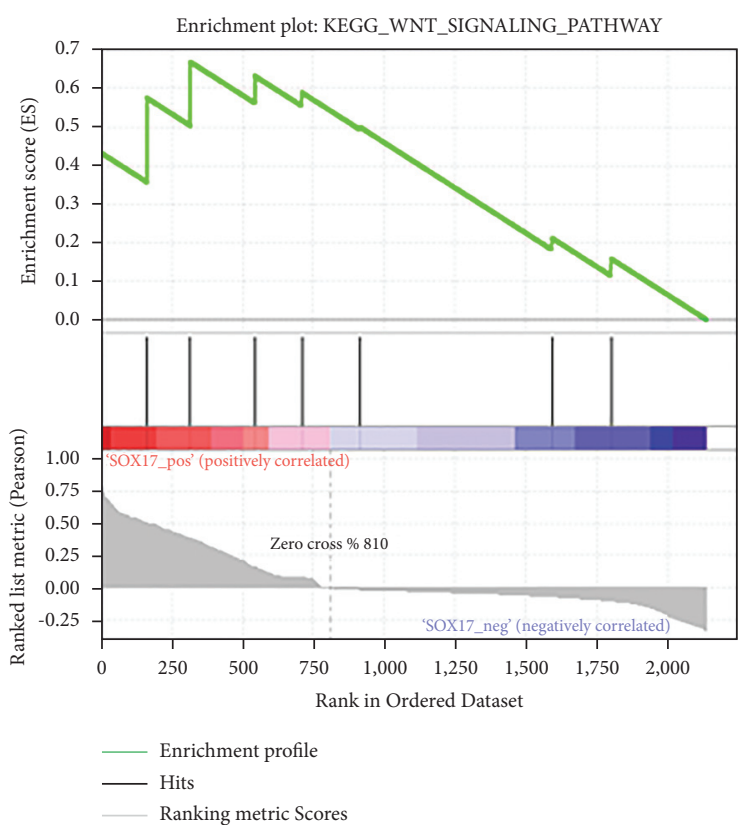

(b)

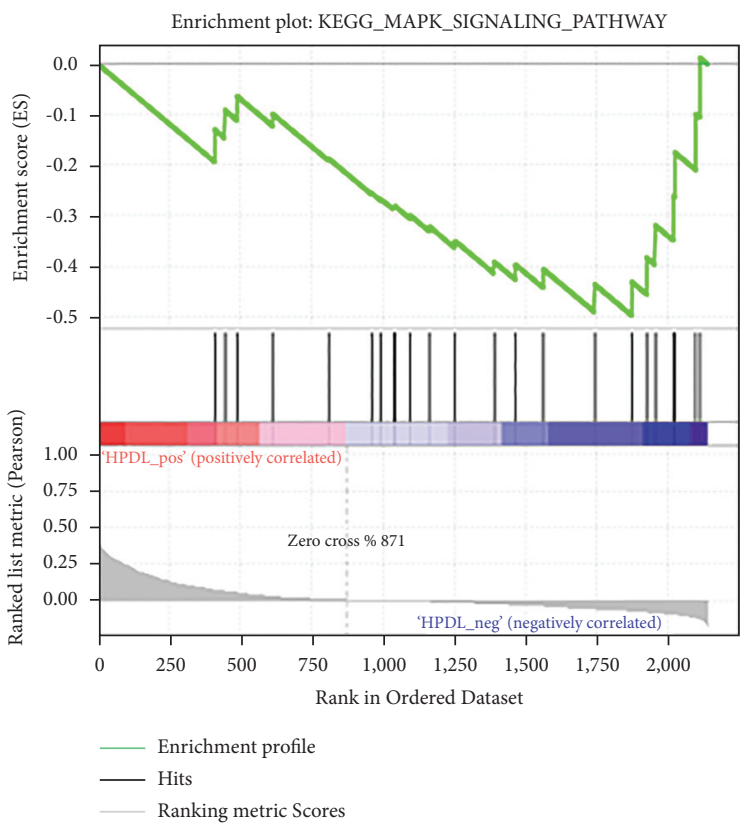

(d)

Figure 4: Related pathways for abnormal expression of key genes in patients.

promoter is highly methylated in primary breast tumors, in CTCs isolated from patients with BRCA, and in corresponding cfDNA samples, which provides new predictive ideas for recurrence and prognosis in patients with operable BRCA and metastatic patients [23, 24]. HPDL may have dioxygenase activity. Previous studies have found that HPDL exhibits differential expression in CNS lymphoma compared with nonprimary central nervous system (CNS) lymphoma [25]. However, understanding the role of HPDL in BRCA needs further research and interpretation, which provides an idea for the in-depth study of the molecular mechanism of BRCA.
Intracellular signaling pathways regulate various cellular activities. We performed GSEA identification on SOX17 and HPDL to further explore the small-molecule regulation mechanism of BRCA and found that signaling pathways with significant changes in enrichment exist between patients with low expression and high expression. When SOX17 is downregulated, the enriched pathways mainly included JAK-STAT signaling pathway and Wnt signaling pathway. It is well known that the JAKSTAT signaling pathway, a signal transduction pathway stimulated by cytokines, is involved in biological processes, such as cell proliferation, differentiation, 
apoptosis, and immune regulation, and is associated with pathogenesis of many tumors, such as liver cancer, ovarian cancer, and BRCA [26-28]. The major cellular processes during BRCA development rely on JAK/STAT signaling to coordinate growth factor function. Previous studies have found that activation of the JAK/STAT pathway is common in triple-negative BRCA, which can affect the expression of genes controlling immune signals. Dysregulated JAK/STAT signaling has been implicated in BRCA metastasis, associated with high risk of recurrence [29-31]. The Wnt signaling pathway plays a crucial role in early embryonic development, organ formation, tissue regeneration, and other physiological processes, often involving stem cell control, which may induce cancer if a key protein is mutated [32]. Wnt signaling pathway involves the onset and treatment of colorectal cancer, pancreatic cancer, gastric cancer, and other tumors [33-35]. Yang et al. confirmed that SOX17 is a target gene of miR-194-5p. In mouse studies, knockdown of miR-194$5 \mathrm{p}$ in BRCA cells may increase SOX17 expression and regulate the signaling pathway of $\mathrm{Wnt} / \beta$-catenin [36]. Therefore, increased expression of SOX17 can activate the Wnt signaling pathway and, thus, participate in the pathogenesis of BRCA. In addition, the enrichment results of SOX17 include pathways related to cell growth, division, and proliferation of oocyte meiosis, ABC transporters, and neuroactive ligand-receptor interaction.

The enrichment pathways of HPDL upregulation mainly include cell cycle and P53 signaling pathway. And the HPDL downregulation is mainly enriched in MAPK signaling pathway and TGF- $\beta$ signaling pathway. Both cell cycle and p53 signaling pathways are involved in cell division and proliferation. The p53 gene is called the "guardian of the genome," but when p53 is deregulated, it participates in the development and proliferation of various tumor cells [37]. Both MAPK and TGF- $\beta$ signaling pathway are involved in cell growth, differentiation, and apoptosis. In recent studies, abnormal activation of the MAPK signaling pathway signal has been found to favor the abnormal proliferation of malignant cells [38]. TGF- $\beta$ signaling acts as suppressor and inducer of tumor progression during the early and late stages of cancer and can trigger a cascade of reactions that mobilize cancer cells $[39,40]$.

Recent studies have demonstrated the consequences of genetic variation in regulating overall risk associated with BRCA patients. In the study so far, we explored the effects of CNV and DNA methylation on gene expression levels and OS of BRCA patients and found that CNV can affect DNA methylation levels. CNV and methylation of SOX17 and HPDL are related to expression and regulation. In addition, the CNV of SOX17 and HPDL were also correlated with methylation levels. In addition, we found methylation sites for SOX17 and HPDL associated with BRCA prognosis. DNA methylation is an effective regulator of gene expression. If the $\mathrm{CpG}$ island is located in the promoter region of a gene, the methylation of the $\mathrm{CpG}$ island will significantly reduce or even completely silence the transcription of the gene and then affect the protein expression. In this study, due to data and conditional restrictions, we did not distinguish whether it was on the promoter or DNA when screening prognostic related methylation sites, which is what we will explore in the next study. Finally, by enriching the low and high expression pathways of SOX17 and HPDL, pathways related to BRCA progression have been discovered, including the JAK-STAT/Wnt/P53/MAPK signaling pathway.

However, this research also has certain limitations. For example, the quality of the samples in the TCGA database is very high, but the number of samples is very large. Therefore, the development of biomarkers for diagnosis and treatment still needs further clinical data verification. In future work, we will further increase the in-depth research and verification of our research results.

\section{Conclusion}

In summary, by comprehensively assessing the effects of $\mathrm{CNV}$ and DNA methylation on gene expression and patient OS, the CNV and DNA methylation associated with the risk of BRCA recurrence and prognosis were identified. These new discoveries are very promising. Prognostic assessment at the genome level may not only be useful for identifying new prognostic biomarkers, but would also open up new horizons for novel pathways involved in BRCA progression, serving the potential goal of developing more effective therapeutic strategies.

\section{Abbreviations \\ BRCA: Breast cancer \\ CNV: Copy number variation \\ TCGA: The Cancer Genome Atlas \\ GSEA: Genetic Set Enrichment Analysis \\ OS: Overall survival.}

\section{Data Availability}

The datasets generated and/or analyzed during the current study are available in the TCGA (https://cancergenome.nih. gov/).

\section{Disclosure}

Chundi Gao and Huayao Li should be considered co-first authors. The manuscript has been submitted as preprint in the following link: https://www.researchsquare.com/article/ rs- $18876 / \mathrm{v} 1$.

\section{Conflicts of Interest}

The authors declare that they have no conflicts of interest regarding the publication of this study.

\section{Authors' Contributions}

CG and HL contributed equally to this work. CG and CS conceived and designed the study. JZ, HL, and JW performed data analysis. CL, CZ, and LL contributed analysis 
tools. CG and HL were major contributors in writing the manuscript. All authors read and approved the final version of manuscript.

\section{Acknowledgments}

This work was supported by grants from the National Natural Science Foundation of China (no. 81973677).

\section{Supplementary Materials}

Supplementary Material 1: genes with abnormal CNV mutations between breast cancer and normal tissues. (Supplementary Materials)

\section{References}

[1] K. Tomczak, P. Czerwińska, and M. Wiznerowicz, "Review the cancer genome Atlas (TCGA): an immeasurable source of knowledge," Współczesna Onkologia, vol. 1A, pp. 68-77, 2015.

[2] H. Kilpinen, S. M. Waszak, A. R. Gschwind et al., "Coordinated effects of sequence variation on DNA binding, chromatin structure, and transcription," Science, vol. 342, no. 6159, pp. 744-747, 2013.

[3] D. Schübeler, "Function and information content of DNA methylation," Nature, vol. 517, no. 7534, pp. 321-326, 2015.

[4] R. Sharma, "Breast cancer incidence, mortality and mortalityto-incidence ratio (MIR) are associated with human development, 1990-2016: evidence from Global Burden of Disease Study 2016," Breast Cancer, vol. 26, no. 4, pp. 428-445, 2019.

[5] B. R. Paolella, W. J. Gibson, L. M. Urbanski et al., "Copynumber and gene dependency analysis reveals partial copy loss of wild-type SF3B1 as a novel cancer vulnerability," ELife, vol. 6, 2017.

[6] A. Sud, B. Kinnersley, and R. S. Houlston, "Genome-wide association studies of cancer: current insights and future perspectives," Nature Reviews Cancer, vol. 17, no. 11, pp. 692-704, 2017.

[7] C. Curtis, S. P. Shah, S. P. Shah et al., "The genomic and transcriptomic architecture of 2,000 breast tumours reveals novel subgroups," Nature, vol. 486, no. 7403, pp. 346-352, 2012.

[8] H.-J. Schulten, M. Bangash, S. Karim et al., "Comprehensive molecular biomarker identification in breast cancer brain metastases," Journal of Translational Medicine, vol. 15, no. 1, p. 269, 2017.

[9] L. Wein and S. Loi, "Mechanisms of resistance of chemotherapy in early-stage triple negative breast cancer (TNBC)," The Breast, vol. 34, no. 1, pp. S27-S30, 2017.

[10] F. Yang, Y. Wang, Q. Li et al., "Intratumor heterogeneity predicts metastasis of triple-negative breast cancer," Carcinogenesis, vol. 38, no. 9, pp. 900-909, 2017.

[11] M. J. Fackler, C. B. Umbricht, D. Williams et al., "Genomewide methylation analysis identifies genes specific to breast cancer hormone receptor status and risk of recurrence," Cancer Research, vol. 71, no. 19, pp. 6195-6207, 2011.

[12] T. Fleischer, A. Frigessi, K. C. Johnson et al., "Genome-wide DNA methylation profiles in progression to in situ and invasive carcinoma of the breast with impact on gene transcription and prognosis," Genome Biology, vol. 15, no. 8, p. $435,2014$.

[13] C. Stirzaker, E. Zotenko, J. Z. Song et al., "Methylome sequencing in triple-negative breast cancer reveals distinct methylation clusters with prognostic value," Nature Communications, vol. 6, no. 1, p. 5899, 2015.

[14] W. Jin, Q.-Z. Li, Y.-C. Zuo et al., "Relationship between DNA methylation in key region and the differential expressions of genes in human breast tumor tissue," DNA and Cell Biology, vol. 38, no. 1, pp. 49-62, 2019.

[15] B. Song, L. Wang, Y. Zhang et al., "Combined detection of HER2, Ki67, and GSTP1 genes on the diagnosis and prognosis of breast cancer," Cancer Biotherapy and Radiopharmaceuticals, vol. 34, no. 2, pp. 85-90, 2019.

[16] A. Shilpi, Y. Bi, S. Jung, S. K. Patra, and R. V. Davuluri, "Identification of genetic and epigenetic variants associated with breast cancer prognosis by integrative bioinformatics analysis," Cancer Informatics, vol. 16, pp. 1-13, 2017.

[17] S. Dietz, A. Lifshitz, D. Kazdal et al., "Global DNA methylation reflects spatial heterogeneity and molecular evolution of lung adenocarcinomas," International Journal of Cancer, vol. 144, no. 5, pp. 1061-1072, 2019.

[18] A. Subramanian, P. Tamayo, V. K. Mootha et al., "Gene set enrichment analysis: a knowledge-based approach for interpreting genome-wide expression profiles," Proceedings of the National Academy of Sciences, vol. 102, no. 43, pp. 1554515550, 2005.

[19] J. Wang, X.-F. Yu, N. OUYang et al., "Multi-platform analysis of methylation-regulated genes in human lung adenocarcinoma," Journal of Toxicology and Environmental Health, Part A, vol. 82, no. 1, pp. 37-45, 2019.

[20] L. Yao, H. Shen, P. W. Laird, P. J. Farnham, and B. P. Berman, "Inferring regulatory element landscapes and transcription factor networks from cancer methylomes," Genome Biology, vol. 16, no. 1, p. 105, 2015.

[21] I. Balgkouranidou, M. Chimonidou, G. Milaki et al., "SOX17 promoter methylation in plasma circulating tumor DNA of patients with non-small cell lung cancer," Clinical Chemistry and Laboratory Medicine, vol. 54, no. 8, pp. 1385-1393, 2016.

[22] D. Fu, C. Ren, H. Tan et al., "Sox17 promoter methylation in plasma DNA is associated with poor survival and can be used as a prognostic factor in breast cancer," Medicine, vol. 94, no. 11, p. e637, 2015.

[23] M. Chimonidou, A. Strati, N. Malamos, V. Georgoulias, and E. S. Lianidou, "SOX17 promoter methylation in circulating tumor cells and matched cell-free DNA isolated from plasma of patients with breast cancer," Clinical Chemistry, vol. 59, no. 1, pp. 270-279, 2013.

[24] M. Chimonidou, A. Strati, A. Tzitzira et al., "DNA methylation of tumor suppressor and metastasis suppressor genes in circulating tumor cells," Clinical Chemistry, vol. 57, no. 8, pp. 1169-1177, 2011.

[25] D. H. Lim, W. S. Kim, S. J. Kim, H. Y. Yoo, and Y. H. Ko, "Microarray gene-expression profiling analysis comparing PCNSL and non-CNS diffuse large B-cell lymphoma," Anticancer Research, vol. 35, pp. 3333-3340, 2015.

[26] Q. Lin, Y.-B. Ling, J.-W. Chen et al., "Circular RNA circCDK13 suppresses cell proliferation, migration and invasion by modulating the JAK/STAT and PI3K/AKT pathways in liver cancer," International Journal of Oncology, vol. 53, pp. 246-256, 2018.

[27] A.-Q. Shang, J. Wu, F. Bi et al., "Relationship between HER2 and JAK/STAT-SOCS3 signaling pathway and clinicopathological features and prognosis of ovarian cancer," Cancer Biology \& Therapy, vol. 18, no. 5, pp. 314-322, 2017.

[28] S. Xu, D. Kong, Q. Chen, Y. Ping, and D. Pang, "Oncogenic long noncoding RNA landscape in breast cancer," Molecular Cancer, vol. 16, no. 1, p. 129, 2017. 
[29] M. Chen, B. Pockaj, M. Andreozzi et al., "JAK2 and PD-L1 amplification enhance the dynamic expression of PD-L1 in triple-negative breast cancer," Clinical Breast Cancer, vol. 18, no. 5, pp. e1205-e1215, 2018.

[30] P. Khanna, J. S. Lee, A. Sereemaspun, H. Lee, and G. H. Baeg, "GRAMD1B regulates cell migration in breast cancer cells through JAK/STAT and AKT signaling," Scientific Reports, vol. 8, no. 1, p. 9511, 2018.

[31] P. D. Rädler, B. L. Wehde, and K.-U. Wagner, "Crosstalk between STAT5 activation and PI3K/AKT functions in normal and transformed mammary epithelial cells," Molecular and Cellular Endocrinology, vol. 451, pp. 31-39, 2017.

[32] A. Banerjee, G. Jothimani, S. V. Prasad, F. Marotta, and S. Pathak, "Targeting Wnt signaling through small molecules in governing stem cell fate and diseases," Endocrine, Metabolic \& Immune Disorders-Drug Targets, vol. 19, no. 3, pp. 233246, 2019.

[33] M. L. Lu, Y. Zhang, J. Li et al., "MicroRNA-124 inhibits colorectal cancer cell proliferation and suppresses tumor growth by interacting with PLCB1 and regulating Wnt/betacatenin signaling pathway," European Review for Medical and Pharmacological Sciences, vol. 23, pp. 121-136, 2019.

[34] S. K. Saha, Y. Yin, H. S. Chae, and S.-G. Cho, "Opposing regulation of cancer properties via KRT19-mediated differential modulation of wnt $/ \beta$-catenin/notch signaling in breast and colon cancers," Cancers, vol. 11, no. 1, p. 99, 2019.

[35] F. Wang, W. Zhu, R. Yang, W. Xie, and D. Wang, "LncRNA ZEB2-AS1 contributes to the tumorigenesis of gastric cancer via activating the Wnt/ $\beta$-catenin pathway," Molecular and Cellular Biochemistry, vol. 456, no. 1-2, pp. 73-83, 2019.

[36] F. Yang, Z. Xiao, and S. Zhang, "Knockdown of miR-194-5p inhibits cell proliferation, migration and invasion in breast cancer by regulating the $\mathrm{Wnt} / \beta$-catenin signaling pathway," International Journal of Molecular Medicine, vol. 42, pp. 3355-3363, 2018.

[37] A. A. Farooqi, M. de la Roche, M. B. A. Djamgoz, and Z. H. Siddik, "Overview of the oncogenic signaling pathways in colorectal cancer: mechanistic insights," Seminars in Cancer Biology, vol. 58, pp. 65-79, 2019.

[38] G. Gampa, M. Kim, A. S. Mohammad et al., "Brain distribution and active efflux of three panRAF inhibitors: considerations in the treatment of melanoma brain metastases," Journal of Pharmacology and Experimental Therapeutics, vol. 368, no. 3, pp. 446-461, 2019.

[39] E. Abedini Bakhshmand and B. M. Soltani, "Regulatory effect of hsa-miR-5590-3P on TGF $\beta$ signaling through targeting of TGF $\beta$-R1, TGF $\beta$-R2, SMAD3 and SMAD4 transcripts," Biological Chemistry, vol. 400, no. 5, pp. 677-685, 2018.

[40] Z. Zhao, D. Hao, L. Wang et al., "CtBP promotes metastasis of breast cancer through repressing cholesterol and activating TGF- $\beta$ signaling," Oncogene, vol. 38, no. 12, pp. 2076-2091, 2018. 